\title{
STUDY OF BEAM-BEAM INTERACTIONS WITH/WITHOUT CROSSING ANGLE
}

\author{
K. Ohmi, M. Tawada and K. Oide \\ KEK, Oho, Tsukuba, 305-0801, Japan
}

\begin{abstract}
In recent high luminosity colliders, the finite crossing angle scheme becomes popular to gain the multiplicity of luminosity with multi-bunch or long bunch operation. Success of KEKB showed that it was no problem to achieve the beam-beam parameter up to 0.05 . We have studied the beam-beam interactions with/without crossing angle toward higher luminosity. We discuss how the crossing angle affects the beam-beam parameter and luminosity in the present KEK B factory (KEKB) using computer simulations.
\end{abstract}

\section{INTRODUCTION}

High luminosity B factories, KEKB and PEP-II are operated successfully at KEK and SLAC, respectively. The collision scheme in KEKB was designed so that two beams collide with a finite crossing angle, $2 \times 11 \mathrm{mrad}$, by $2 \mathrm{~ns}$ repetition. While head-on collision scheme was adopted in PEP-II by 4 ns repetition. Crossing angle makes easy a design of interaction region for the narrow bunch spacing. However the crossing collision scheme had been considered to be a taboo, since the unsuccessful experience of DORIS in DESY. In KEK, many studies were performed to decide the adoption of the crossing collision scheme $[1,2,3]$. KEKB and PEP-II have achieved luminosities of $1.06 \times 10^{34}$ and $0.61 \times 10^{34} \mathrm{~cm}^{-2} \mathrm{~s}^{-1}$, respectively at May of 2003. Such high luminosities were not believed to be realized when their design works had started. In recent high luminosity colliders, the crossing angle scheme becomes popular to gain the high repetition of the luminosity.

The luminosities are achieved by a high repetition frequency, which is $1 / 3 \sim 1 / 4$ of the design. The bunches spacing, which is inverse of the repetition frequency, is 8-6 ns and $6 \mathrm{~ns}$ for KEKB and PEP-II, respectively. Narrower bunch spacing does not contribute the luminosity perhaps due to the electron cloud effect in both of the machines. The operating tune is just slightly upper of half integer in horizontal, and is optimized around 0.54-0.56 in vertical.

We review the crossing collision scheme in the point of view of progress toward higher luminosity.

\section{FORMALISM OF COLLISION WITH CROSSING ANGLE}

We discuss the beam-beam effects with/without crossing angle using computer simulations. The collision with crossing angle is treated by Lorenz boost to a head-on frame from the laboratory frame $[1,2]$ : i.e., particles in the beam are transferred to the head on frame, experience the collision, and are transferred to laboratory frame by the inverse of the Lorenz boost. The transformation is given for a half crossing angle $\theta$ by

$$
\begin{aligned}
x^{*} & =\tan \theta z+\left(1+\frac{p_{x}^{*}}{p_{s}^{*}} \sin \theta\right) x \\
y^{*} & =y+\sin \theta \frac{p_{y}^{*}}{p_{s}^{*}} x \\
z^{*} & =\frac{z}{\cos \theta}-\frac{H^{*}}{p_{s}^{*}} \sin \theta x \\
p_{x}^{*} & =\frac{p_{x}-\tan \theta H}{\cos \theta} \\
p_{y}^{*} & =\frac{p_{y}}{\cos \theta} \\
p_{z}^{*} & =p_{z}-\tan \theta p_{x}+\tan ^{2} \theta H,
\end{aligned}
$$

where

$$
\begin{gathered}
H=\left(1+p_{z}\right)-\sqrt{\left(1+p_{z}\right)^{2}-p_{x}^{2}-p_{y}^{2}} \\
p_{s}=\sqrt{\left(1+p_{z}\right)^{2}-p_{x}^{2}-p_{y}^{2}}
\end{gathered}
$$

A star designates a dynamical variable in the head-on frame. Note that the $x^{*}$ and $y^{*}$ axes are defined in the same direction for both beams, while the $s^{*}$ axis is defined in opposite directions, since the two beams travel in opposite directions.

The linear part of the transformation is expressed by a matrix

$$
M_{\text {crs }}=\left(\begin{array}{cccccc}
1 & 0 & 0 & 0 & \tan \theta & 0 \\
0 & 1 / \cos \theta & 0 & 0 & 0 & 0 \\
0 & 0 & 1 & 0 & 0 & 0 \\
0 & 0 & 0 & 1 / \cos \theta & 0 & 0 \\
0 & 0 & 0 & 0 & 1 / \cos \theta & 0 \\
0 & \tan \theta & 0 & 0 & 0 & 1
\end{array}\right) .
$$

This transformation is not symplectic: the determinant of the Jacobian matrix of the transformation is not 1, but $\cos ^{-3} \theta$. The fact is due to that Lorenz transformation is not symplectic for the accelerator coordinate, because the Hamiltonian is divided by a reference momentum.

The transformation and its inverse are performed before and after the beam-beam interaction, respectively. If we consider only linear part, the revolution matrix is expressed by

$$
M_{c r s} M_{a r c} M_{c r s}^{-1} \text {. }
$$

where $M_{\text {arc }}$ is the transfer matrix of arc section. For small $\theta$, the revolution matrix is equivalent to that with $\mathrm{z}-$ dependent dispersion $\zeta_{x}=\theta$ [4] at the interaction point, 
$s=s^{*}$. The beam envelope has $\langle x z\rangle \propto \zeta_{x}(s)$ in the headon frame.

Crab cavities create a z-dependent dispersion $\zeta_{x, c r a b}(s)$. Controlling $\zeta_{x, c r a b}\left(s^{*}\right)$ at the interaction point using the crab cavities, the effective crossing angle, which the beam experiences, can be chosen arbitrary. Head-on collision of the beams with $\langle x z\rangle=0$ is realized by $\zeta_{x, \text { crab }}=-\theta$ effectively.

We use two simulation models, weak strong and strongstrong models, to study the beam-beam interactions. In the weak-strong model, one beam is fixed Gaussian distribution in the six dimensional phase space, while the other beam is represented by macro-particles. Macro-particles are transferred from the laboratory frame to the head-on one using Eq.(2). The beam envelope of the strong beam is transferred by

$$
M_{c r s}\left\langle\boldsymbol{x} \boldsymbol{x}^{t}\right\rangle M_{c r s}^{t} .
$$

In the strong-strong model, both of the beams are represented by macro-particles, which can have an arbitrary distribution. The beam-beam force is estimated by the particle in cell method [5]. Macro-particles of the both beams are transferred by Eq.(2).

We now consider collision of two beams in the head-on frame. The beam has longitudinal structure, bunch length, The simulation has to take into account of the longitudinal dynamics: bunch length and synchrotron motion. In the weak-strong simulation, we obeyed a method written in Ref. [6], so-called the synchro-beam map. The target bunch, which is 6-D fixed Gaussian distribution, is divided into several longitudinal slices, and collision between a slice and particle in the weak beam is calculated. The collision point of a slice $\left(z_{j}\right)$ and the particle $(z)$ is

$$
s=z-z_{j}
$$

Beam envelope of the slice is transferred to the collision point by

$$
R(s(z))=T(s(z), 0) R(0) T^{t}(s(z), 0) .
$$

Particles are translated

$\boldsymbol{x}(s(z))=\mathcal{T}(s(z)) \boldsymbol{x}(0)=\exp \left[-: \frac{\left(p_{x}^{2}+p_{y}^{2}\right) s(z)}{2}:\right] \boldsymbol{x}(0)$

The beam envelope is a function of $z$, therefore the beambeam force have a longitudinal kick.

In the strong-strong simulation, similar method is used [7]. If we estimate collision between slice by slice at $s=\left(z_{i}-z_{j}\right) / 2,20-30$ slices are needed depending on the beam intensity and the computation time is too long $[8,9,10]$. Two dimensional potential, which determines the beam-beam force, is estimated at a collision point front $\left(s_{f}=\left(z_{i, f}-z_{j}\right) / 2\right)$ and back $\left(s_{b}=\left(z_{i, b}-z_{j}\right) / 2\right)$ face of the $(i$-th) slice containing the particle. The potential of the target slice $\phi(s)$ is transferred to the collision point of the particle by a linear interpolation,

$$
\phi_{j}(s)=\frac{\phi_{j}\left(s_{f}\right)-\phi_{j}\left(s_{b}\right)}{s_{f}-s_{b}}\left(s-s_{b}\right) .
$$

An energy change proportional to $\partial \phi / \partial z$, which is caused by the interaction depending on $z$, is included in the simulation.

We divided a bunch into 5 to 10 slices. The number of slice required depends on the beam-beam parameter: i.e., higher beam-beam parameter requires more number of slices. In our parameter region, the simulation results converged the number of slice.

\section{SIMULATION RESULTS}

We studied the effect of crossing angle on the beambeam parameter and luminosity for the machine parameters as is shown in Table 1. In the weak-strong simulation, 100 macro-particles are tracked up to 40,000 turns $(10 \tau)$, and the luminosity was calculated by averaging it during 20,000 through 40,000 turns. In the strong-strong simulation, 100,000 macro-particles are tracked up to 20,000 turns. The luminosity was given by that calculated at the last turn. The bunch population of electrons $\left(N_{-}\right)$in the high energy ring (HER) is scanned $1 \times 10^{10}$ to $1.2 \times 10^{11}$. The transparency condition was kept as $N_{+} \gamma_{+}=N_{-} \gamma_{-}$to avoid complex behavior for unbalance of the beams. The beam-beam parameter was calculated by the luminosity,

$$
\xi_{y}=\frac{2 r_{e} \beta_{y}}{N_{ \pm} \gamma_{ \pm}} \frac{L}{f_{r e p}}
$$

where the reduction factor of the luminosity and the beambeam parameter is approximated to be the same.

Table 1: Basic parameters of KEKB

\begin{tabular}{|c|c|}
\hline & HER $\quad$ LER \\
\hline$C$ & $3016 m$ \\
$E$ & $8 \mathrm{GeV} \quad 3.5 \mathrm{GeV}$ \\
$\beta_{x} / \beta_{y}$ & $60 \mathrm{~cm} / 7 \mathrm{~mm}$ \\
$\varepsilon_{x} / \varepsilon_{y}$ & $18 \mathrm{~nm} / 0.18 \mathrm{~nm}$ \\
$\sigma_{z}$ & $7 . \mathrm{mm}$ \\
$\nu_{x} / \nu_{y} / \nu_{s}$ & $0.515 / 0.565 / 0.02$ \\
$\tau_{x y} / T_{0}$ & 4,000 turn 4,000 turn \\
$\theta_{c}$ & $0 \sim 2 \times 11 \mathrm{mrad}$ \\
\hline
\end{tabular}

Fig. 1 shows the relation of the beam-beam parameter $\xi$ and the bunch population for head-on and crossing collision with a half crossing angle of $11 \mathrm{mrad}$. The result of the head-on collision is considered to be the same as that of the crossing collision with crab cavities set as $\zeta_{x, c r s}=-\phi$. Pictures (a) and (b) were obtained by the weak-strong and strong-strong simulations, respectively. The beam-beam parameters for the head-on collision is remarkable higher than that for the crossing collision in both of the simulation. The beam-beam parameter $\xi$ is linearly increase up to over 0.2 in the weak strong simulation, while $\xi$ is saturated around 0.1-0.12 in the strong-strong simulation. The beam-beam limit is 0.1 or $>0.2$ for the head-on collision. There is somewhat difference of the beam-beam limit 
for the weak-strong and the strong-strong simulation. $\xi$ at crossing collision is similar behavior for the both of two simulations: that is, $\xi$ is saturated around 0.06 .
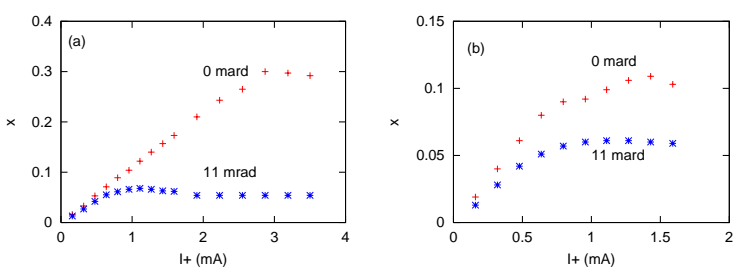

Figure 1: Beam-beam parameters obtained by weak-strong and strong-strong simulations.

Fig. 2 shows luminosity for various crossing angle. The geometrical luminosity, which is also plotted in the figure, has loose dependence for the crossing angle. The simulated luminosity is peak structure near zero-crossing angle for the simulations. The peak structure of the strong-strong simulation is narrower than that of the weak-strong simulation.

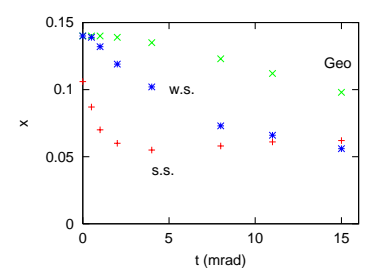

Figure 2: Beam-beam parameters v.s. crossing angle. Three points,

The horizontal tune is chosen to be slightly upper of a half integer in KEKB. The beam-beam behavior depends on the tune value. Fig. 3 shows beam-beam parameter for various horizontal tune given by the weak-strong simulation. The beam-beam parameter decreases for separating from the half integer. The figure shows that the extremely high beam-beam parameter $\sim 0.2$ is achieved at the tune closed to a half integer.

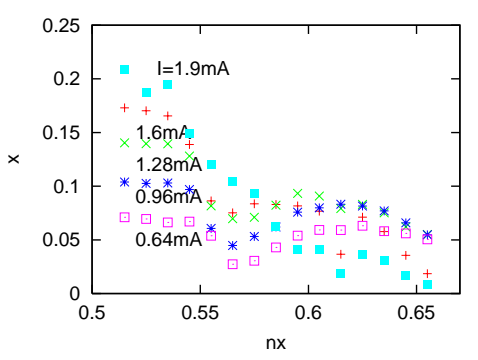

Figure 3: Beam-beam parameter v.s. horizontal tune for various positron current at $\nu_{y}=0.565$.

\section{CONCLUSION}

We studied beam-beam effect with/without crossing angle using weak-strong and strong-strong simulations. The beam-beam parameter $(\xi)$ linearly increased more than 0.2 for the current in the weak-strong simulation and was saturated at $\sim 0.1$ in the strong-strong simulation in the headon collision. While $\xi$ was saturated at around $\sim 0.06$ for a finite crossing angle in both of the simulations. In either case, the beam-beam parameter for the head-on collision is much better than that with the crossing angle. Crab cavities, which realize the head-on collision effectively, can be expected to upgrade the luminosity twice or four times.

The luminosity is degraded by various reasons, for example, orbit and optics errors [11]. The crossing angle is a kind of optics error, $\mathrm{z}$ dependent dispersion $(\zeta)$, at the collision point. When a source which degrades the luminosity exists, the two type of simulations, weak-strong and strong-strong, give similar results.

We have to know the beam-beam parameters for the case that all of sources of degradation are removed. There is difference for the beam-beam limit between the two type of simulations. We do not know whether the weak-strong model is reliable to estimate the beam-beam limit, while unphysical numerical noises, which are contained in the PIC algorithm or longitudinal slicing, may degrade for the strong-strong simulation at a high current. Tune operating point is essential for the high beam-beam parameter. We do not discard the possibility of the very high beam-beam parameter, $\sim 0.2$, obtained by the weak-strong simulation, though more studies are required.

The authors thank members of KEKB commissioning group for fruitful discussions.

\section{REFERENCES}

[1] K. Oide, K. Yokoya, Phys. Rev. A40, 315 (1989).

[2] K. Hirata, Phys. Rev. Lett., 74, 2228 (1995).

[3] “KEKB B-factory design report”, KEK Report 95-7 (1995).

[4] K. Ohmi, K. Hirata and K. Oide, Phys. Rev. E49, 751 (1994).

[5] K. Ohmi, Phys. Rev. E62, 7287 (2000).

[6] K. Hirata, H. Moshammer and F. Ruggiero, Particle Accelerators, 40, 205 (1993).

[7] K. Ohmi and A. W. Chao, proceedings of the MiniWorkshop on Electron-Cloud Simulations for Proton and Positron Beams, CERN, 15-18 April, 2002, CERN-2002001 (2002).

[8] M. Tawada et. al., Proceedings of a Workshop on beambeam effects in circular colliders, Fermilab, June 25-27, 2001, FERMILAB-Conf-01/390-T, 17 (2001).

[9] E. B.Anderson and J. T.Rogers, Proceedings of a Workshop on beam-beam effects in circular colliders, Fermilab, June 25-27, 2001, FERMILAB-Conf-01/390-T, 136 (2001).

[10] Y. Cai, private communications.

[11] K. Ohmi, Proceedings of EPAC2000, 433 (2000). 